\title{
A Case of Eclampsia with Two Special Details of Treatment.*
}

By C. Nepean Longridge, M.D. (Vict.), F.R.C.S. (Eng.), M.R.C.P. (Lond.), late Resident Medical Officer at Queen Charlotte's Hospital.

A FAIR woman, aged 25, and somewhat nervous about herself, was seen in the 0.P. Department of Queen Charlotte's Hospital in the fourth month of her second pregnancy. She had had eclampsia during her first labour, the infant being still born. The urine was free from albumen, there was no ædema, and no symptom of the toxæmia of pregnancy. Vomiting had been slight during the second month. The blood pressure in the right brachial artery taken by the Riva Rocci apparatus was $140 \mathrm{~mm}$. of $\mathrm{Hg}$. She was seen at intervals during the pregnancy, and the urine on all occasions was found to be free from albumen and the general condition good. For the last month she was sent to a home where she could be under supervision. On August 31 she complained of headache, slight vomiting and diarrhoea (15 actions). At 10-30 p.m. she was brought to the hospital; immediately on admission she had a fit and became unconscious. Two fits followed in rapid succession, the duration of each being about three minutes. The size of the uterus corresponded to that of a full-time pregnancy, the fotal heart was heard and the child was lying in the L.O.A. position with the head well engaged. The cervix was short and admitted two fingers, the membranes being unruptured. The blood pressure was $160 \mathrm{~mm}$. of $\mathrm{Hg}$., the urine became nearly solid on boiling; there was some cedema of the feet and legs.

Five grains of calomel and $\frac{1}{4}$ grain of morphia were given. The calomel was vomited at once, and replaced in about half an hour by an ounce of oil. She had four more fits between one and three o'clock, when another $\frac{1}{4}$ grain of morphia was given. There was marked cyanosis during the fits, and deep unconsciousness between them. At 6-30 a.m. the os was found to be fully dilated, the membranes were therefore ruptured, forceps applied, and the child delivered. No attempt was made to check bleeding, which amounted to 12 ounces. She had a fit during the birth of the placenta. From 7-10-30 a.m. she had six fits. A third injection of morphia was

* Read at the Obstetrical Society of London, November, 1905. 
given at 9-30. From 10-30 to 11-30 four fits occurred of greater severity and duration than any which had hitherto taken place. $\Delta_{s}$ the patient's condition appeared to be getting rapidly worse, the right median basilic vein was opened and about 10 ounces of blood removed and 32 ounces of saline run into the vein. The stomach was washed out with warm water, half a pint of saline, 5 ounces of milk, and an ounce of oil being left in. In the next two hours three fits occurred. The bowels being not yet open, an enema containing an ounce of magnesium sulphate and half an ounce of glycerine was given, together with $2 \mathrm{~m}$. of croton oil by the mouth; there were two fits in the next hour. She was then given occasional whiffs of chloroform, being kept warmly wrapped up with hot bottles and blankets. At 3 o'clock profuse perspiration began and continued, and some water was passed. Two more slight fits were noticed about two hours later. At 6-30 as the pulse was very rapid and feeble, a fourth injection of morphia was given, and two pints of saline with an ounce of brandy by the bowel. The patient soon improved and became less unconscious, and was able to swallow half a pint of albumen water. At 10-40 p.m. the bowels acted for the first time to the accompaniment of a slight fit, this being the 25th and the last. During the day the redema increased rapidly, the hands and face being particularly affected. The pupils remained firmly contracted, except during the fits, when they dilated. During the night she was restless, took plenty of sweetened milk and barley water, and passed urine. Ten watery foul-smelling motions were passed. The temperature which, at the cessation of the fits, was $105^{\circ}$, fell in the morning. There were no pulmonary complications. The patient remained unconscious from 10-30 p.m. on Aug. 31st until about 6 a.m. on Sept. 3rd. She suffered from a marked loss of memory, which was not completely restored six weeks after her illness. On Sept. 4th she developed jaundice which lasted until Sept. 9th; there were no hæmorrhages. At the same time she had some labial herpes and itching of the head. On Sept. 4th the cedema had practically disappeared and the blood pressure was reduced to $120 \mathrm{~mm}$. Hg. The urine contained about $\frac{1}{3}$ albumen and a large quantity of casts. The albumen had disappeared by the sixth day. Subsequent progress was uneventful and the infant did well.

The day after delivery the alkalinity of the blood was examined by Wright' ${ }^{1}$ method and found to be equal to $\frac{\mathrm{N}}{75} \mathrm{H}_{2} \mathrm{SO}_{4}$ the alkalinity of healthy blood being $\frac{\mathrm{N}}{35} \mathrm{H}_{2} \mathrm{SO}_{4}$. A mixture containing magnesium sulphate one ounce, citrate of soda half a drachm, and liquid extract of 
cascara half a drachm, was given every three hours. A pint of saline containing an ounce of lactose was injected into the rectum every four hours. Citrate of soda was given to increase the alkalinity of the blood. In two days the alkalinity was brought up to $\frac{\mathrm{N}}{30} \mathrm{H}_{2} \mathrm{SO}_{4}$. The first estimation of the alkalinity was only made twelve hours after the fits had ceased, and a considerable elimination of toxin had taken place. In another mild case of eclampsia where the patient had four fits the alkalinity was found to be $\frac{N}{80} \mathrm{H}_{2} \mathrm{SO}_{4}$, while the fits were occurring. A third case gave an alkalinity of $\frac{N}{60}$. This detail may be an important one as showing that the intoxication, whatever it is, is probably an acid one, and as an indication for the exhibition of alkalies. A citrate was given as it is absorbed readily into the blood as a carbonate, and so increases the alkalinity. Jardine, ${ }^{2}$ a strong advocate for transfusion in these cases, has recommended the addition of one drachm of sodium acetate to the pint of normal saline for transfusion, with a view to securing diuresis, and not with the intention of increasing the alkalinity of the blood, but it is possible that his excellent results are partly due to the increased alkalinity induced by his method.

A second detail of treatment rests on a highly theoretical basis. The patient was given considerable quantities of sugar by the mouth and rectum. None appeared in the urine. The patient became markedly jaundiced, showing that the liver had gone through a period of stress, and one imagines that the bile canaliculi became blocked with the débris of the storm, in much the same way that the tubules of the kidney became choked with casts. The work of Schiff, Bouchard and Roger ${ }^{3}$ has proved that the liver possesses an antitoxic function. It seems that this antitoxic function can only be exercised in the presence of glycogen, and further that the power of the liver to decrease the toxicity of poisons depends directly on the amount of glycogen contained in its cells. It was thought in this case that in consequence of the high temperature, and the severe muscular exertion which the patient had undergone, the glycogenic content of the liver was practically exhausted, and sugar was given with a view of increasing it, and so increasing the antitoxic power of the liver. Three years ago the author advocated the use of glucose in feeding typhoid patients on these grounds, 4 and his small experience as a House Physician led him to regard the results with favour.

It is probable that transfusion saved the life of this patient, and the case illustrates the arguments urged by Jardine in favour of this 
line of treatment. Of the twenty-five fits, seven occurred before, one during, and seventeen after, delivery. Ten of these seventeen occurred in five hours before transfusion and were increasing in severity. Seven occurred in ten and a half hours after transfusion, the last three being slight in character. It is of course impossible to say how far the two details of the after treatment which have been described contributed to the rapid recovery of the patient, but they appear to rest upon a thoroughly scientific basis.

The author is indebted to Dr. Griffith for permission to publish this case.

\section{Rerferences.}

1. Lancet, September 18th, 1897, p. 719.

2. Brit. Journal of Obstet, and Gynacol., July, 1905.

3. La Presse Medicale, June 26th, 1897.

4. B.M.J., Angust 30th, 1902, p. 616 . 